\title{
INTEGRAL IMAGE TUBE-OPTICAL SYSTEMS \\ FOR THE FAR UV
}

\author{
GUIDO CHINCARIN I
}

NASA Manned Spacecraft Center, Houston, Tex. and Wesleyan University, Middletown, Conn., U.S.A.

An important feature of observations made outside the atmosphere from manned or unmanned spacecrafts is the reliability and ease of operation, speed and accuracy in the collection of data. A good compromise including the above features is the development of image tube techniques in skilfully matching optics and electronics.

For narrow band direct photography (or objective grating spectra) we plan to use an $f / 1.5$ Schmidt Camera with a photocathode located on the focal surface (see

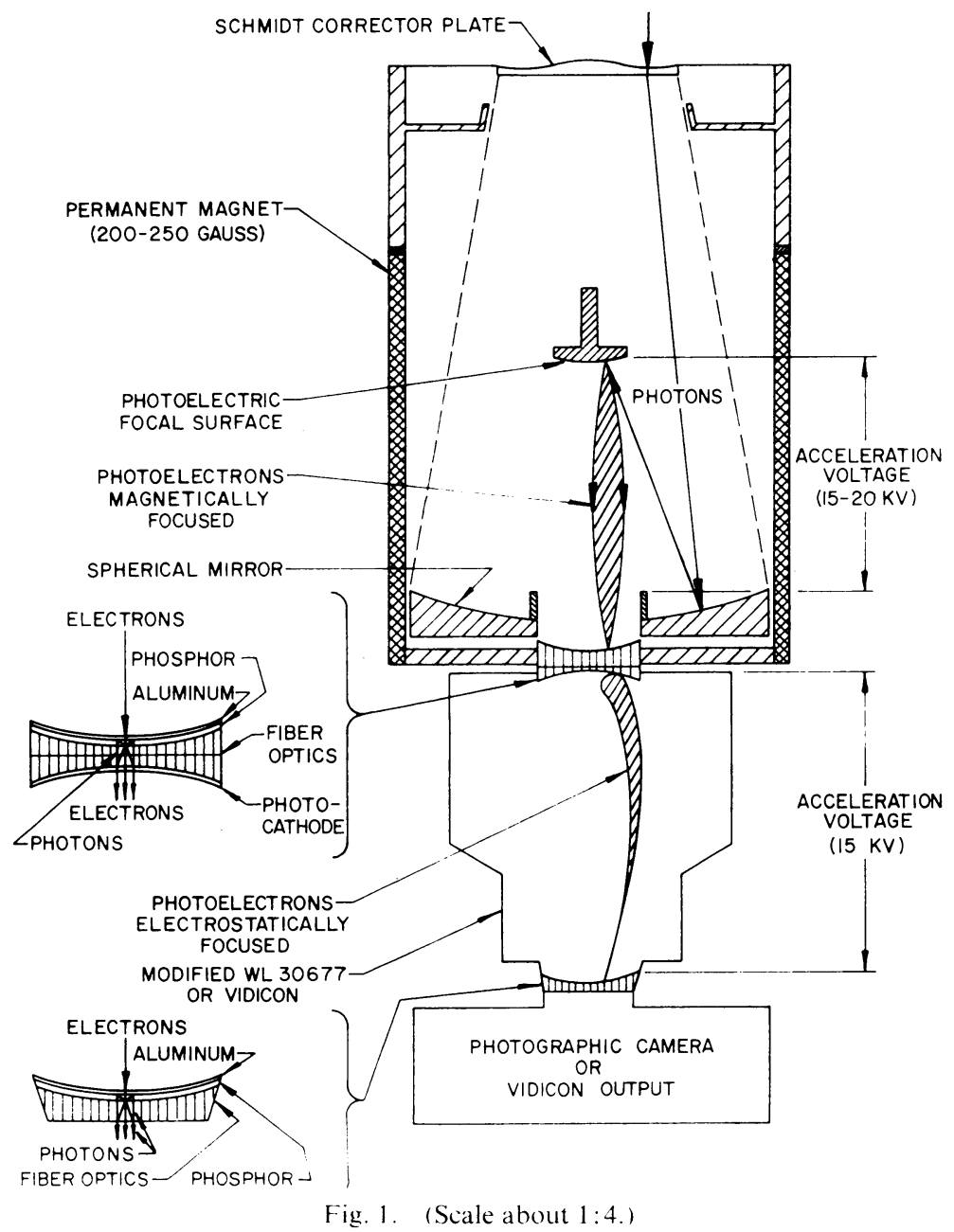


Figure 1). The photoelectrons are accelerated and magnetically focused onto a phosphor-fiber optics surface. The curvature of this surface will partially correct for distortions of the electric field introduced by the curved photocathode. The second stage can be any other tube, electrostatically or magnetically focused, having a fiber optics faceplate butted to the first stage.

From this design, one can generate a family of astronomical instruments with various focal ratios and a variety of passbands choosing various combinations of photocathodes and correcting plates. In the range from 2000 to 3500 , the use of filters is not very difficult. The expected resolution of the system, using six (6) micron fiber optics, is about $25 \mathrm{lp} / \mathrm{mm}$ or better. The gain of the camera depends on wavelength. Use of an opaque photocathode (e.g. about $40 \%$ efficiency for CsI at $\lambda=1500$ ), and of a phosphor as a photon producer yields an expected photon gain of about 2500 (output photons per incident photon). Of course, the speed and/or limiting magnitude is determined largely by the focal ratio of the optics and the demagnification factor of the second stage used as in Figure 1. However, the camera described above is not very suitable for very wide passbands such as is required for a camera in a UV spectrograph. This is due to the fact that all available materials for the UV radiation have an index of refraction which changes very rapidly with wavelength in the UV and introduces optical aberrations. For broad bandpass, therefore, another optical approach should be used.

Following some suggestions of Karl Henize, we can utilize all reflecting Schmidt optics as designed by L. C. Epstein (Sky and Telescope, Vol. 33, April 1967). Figure 2 is a sketch of a spectrograph with an $f / 3$ all reflecting Schmidt image tube camera.

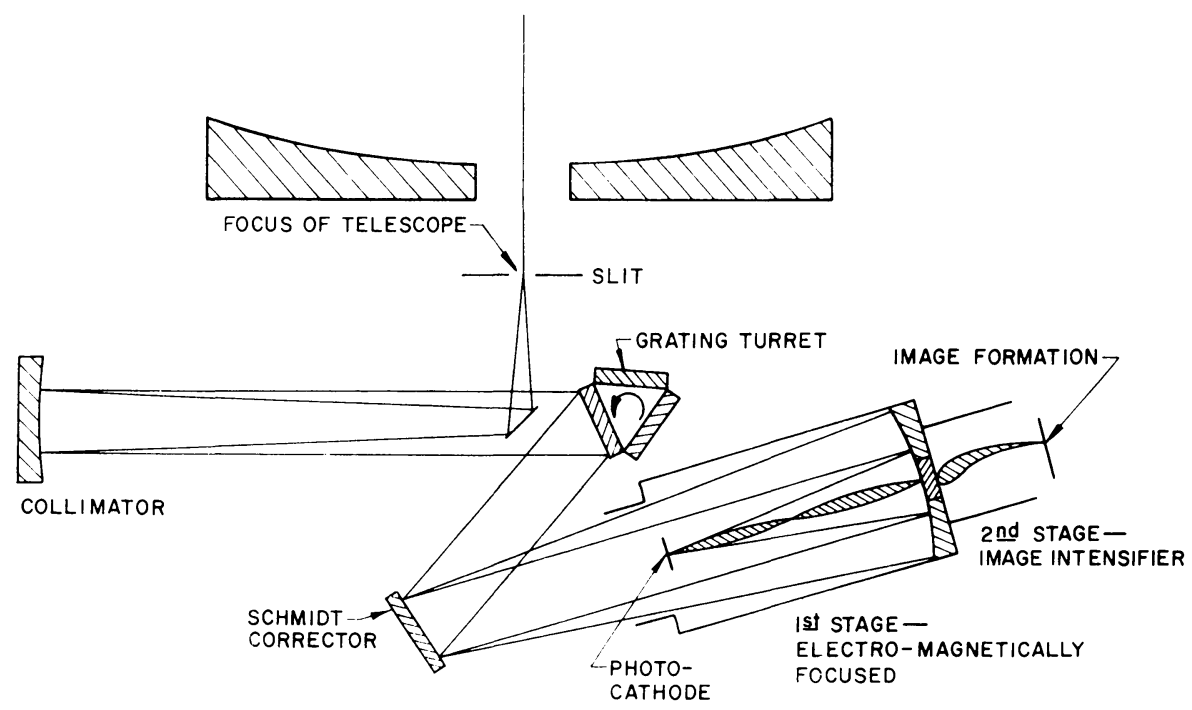

CAMERA $\equiv$ INTEGRAL IMAGE TUBE-ALL REFLECTING SCHMIDT SYSTEM

Fig. 2. 
Systems as fast as $f / 2$ should be possible without losing resolution. We are usually limited by the various optical-electronic adjustments and by the fiber optics. With a $70 \mathrm{~mm}$ collimator and a high dispersion grating $(3600$ grooves $/ \mathrm{mm})$, we can obtain first order resolution better than $0.5 \AA$. With 1200 grooves $/ \mathrm{mm}$, the spectral range is about $700 \AA$. The resolution is $1.6 \AA$ and it should be possible to reach (density on plate 0.1 ) an $18 \mathrm{mag} B$ star in the UV with a $45 \mathrm{~cm}$ telescope and a 30-min exposure widening the spectrum to $0.4 \mathrm{~mm}$. Other classical combinations are possible, however, details depend on where we are going to use it. Magnetic fields of about 200-300 G and an accelerating voltage of about $20000 \mathrm{~V}$ are required. Even without a detailed analysis, the gain of such equipment is quite straightforward and certainly suitable for observations of faint extended objects.

I would like to thank Dr T. L. Page and Dr Y. Kondo for suggesting the possibility of using image tube techniques in space observations. The work is supported by the National Aeronautics and Space Administration.

\section{Reference}

Epstein, L. C.: 1967, Sky Telesc. 33, April.

\section{DISCUSSION}

J.W. Campbell: What is the size of the lithium fluoride corrector plate?

G. Chincarini: The instrument shown here has a diameter of about $17 \mathrm{~cm}$. The radius of the corrector is about $8 \mathrm{~cm}$. 